\title{
Lymphatic microvessel density using D2-40 is associated with nodal metastasis in non-small cell lung cancer
}

\author{
IWAO TAKANAMI \\ Department of Surgery, Teikyo University School of Medicine, Tokyo, Japan
}

Received September 6, 2005; Accepted October 28, 2005

\begin{abstract}
The monoclonal antibody D2-40 is a new selective marker for lymphatic endothelium. The lymphatic microvessel density (LMVD) using D2-40 has not yet been evaluated in non-small cell lung cancer (NSCLC). The aim of this study was to evaluate LMVD using D2-40 in NSCLC. We investigated LMVD in 77 patients with NSCLC who underwent curative tumor resection. We also determined the relation between LMVD and clinicopathologic factors, VEGF-C and Ang-2 and microvessel density (MVD) using factor VIII-related antigen. The median number of D2-40positive vessels in the highest LMVD was 25 (range, 5-71). LMVD was significantly associated with tumor status, lymph node metastasis, stage, lymphatic invasion, VEGF-C protein and MVD ( $\mathrm{p}=0.0149$ for tumor status; $\mathrm{p}<0.0001$ for nodal status; $p<0.0001$ for stage; $p=0.0153$ for lymphatic invasion; $\mathrm{p}=0.0030$ for VEGF-C, and $\mathrm{p}=0.0029$ for MVD). Furthermore, LMVD using D2-40 expression was shown to be an independent predictor of lymph node metastasis by multivariate analysis $(\mathrm{p}=0.0070)$. These data indicate that a high LMVD by D2-40 may be an indicator of lymph node metastasis in NSCLC.
\end{abstract}

\section{Introduction}

Cancer cell spread to regional lymph nodes is an early event in the progression of many solid tumors, and the lymphatic vasculature serves as the primary route for metastatic spread. Lymphangiogenesis studies have been limited by lack of specific lymphatic endothelial markers. Monoclonal antibody D2-40 was reported to be a selective marker for lymphatic

Correspondence to: Dr Iwao Takanami, Department of Surgery, Teikyo University School of Medicine, 11-1 Kaga 2-Chome, Itabashi-Ku, Tokyo 173-8605, Japan

E-mail: takanami@med.teikyo-u.ac.jp

Abbreviations: Ang-2, angiopoietin-2; NSCLC, non-small cell lung cancer; LMVD, lymphatic microvessel density; MVD, microvessel density

Key words: D2-40, immunohistochemistry, non-small cell lung cancer, lymph node metastasis, lymphangiogenesis endothelium (1), and has been shown to be useful in identifying the presence of lymphatic invasion in various malignant neoplasms (2). Studies of various tumors have shown the potential clinical significance of lymphangiogenesis, suggesting that lymphatic microvessel density correlates with tumor growth and metastasis (3). The modulation of lymphatic vascular development has been identified, and vascular endothelial growth factor-C (VEGF-C) (4), angiopoietin-2 (Ang-2) (5) and angiogenesis (6) are reported to regulate lymphatic development. The lymphatic microvessel density using D2-40 has not yet been evaluated in non-small cell lung cancer (NSCLC), and it remains to be elucidated whether the lymphatic microvessel density is related to VEGF-C, Ang-2 and blood microvessel density in NSCLC. Thus, we investigated the lymphatic microvessel density using immunohistochemical D2-40 in a series of 77 cases of curatively resected NSCLC for its clinical significance. We also determined whether the lymphatic microvessel density using D2-40 correlates with the immunohistochemical assay of VEGF-C, Ang-2 and blood microvessel density using factor VIII-related antigen (F8RA).

\section{Materials and methods}

Patients and tumors. Tumor tissue was collected from 77 patients with NSCLC who underwent curative surgery between 1995 and 1999 at the Department of Surgery, Teikyo University School of Medicine. Patients who died within a month after surgery and patients with a past history of another cancer were excluded from the study. Patients were also excluded if they had received neoadjuvant chemotherapy or radiotherapy. The 77 patients included 56 men and 21 women ranging in age between 34 and 84 years (mean, 66.3 years). With regard to histological type, 43 were adenocarcinomas, 31 were squamous cell carcinomas and 3 were large cell lung carcinomas. There were 21 patients with stage IA, 17 patients with stage IB, 1 patient with stage IIA, 14 patients with stage IIB and 24 patients with stage IIIA. Tissue samples to be used for immunohistochemistry and $\mathrm{H} \& \mathrm{E}$ staining were fixed in formalin and paraffin embedded.

Immunohistochemical staining. Immunohistochemical studies of D2-40, F8RA, VEGF-C and Ang-2 were performed, and these specimens were immunostained using formalin-fixed, paraffin-embedded tissues. The sections were immersed for 30 min in $0.3 \% \mathrm{H}_{2} \mathrm{O}_{2}$ in absolute methanol and treated with 


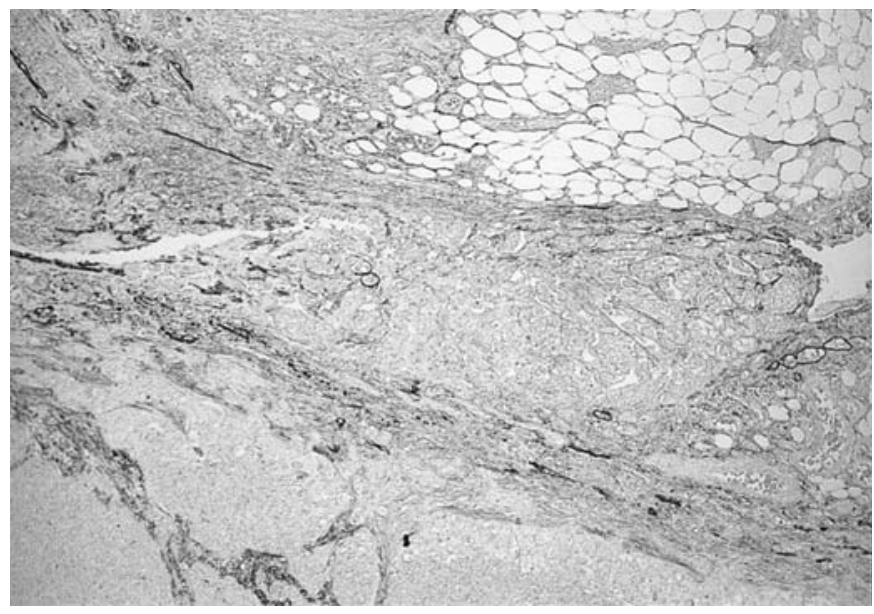

Figure 1. Some D2-40-positive lymphatic vessels are present at the tumor periphery. Original magnification, $\mathrm{x} 15$.

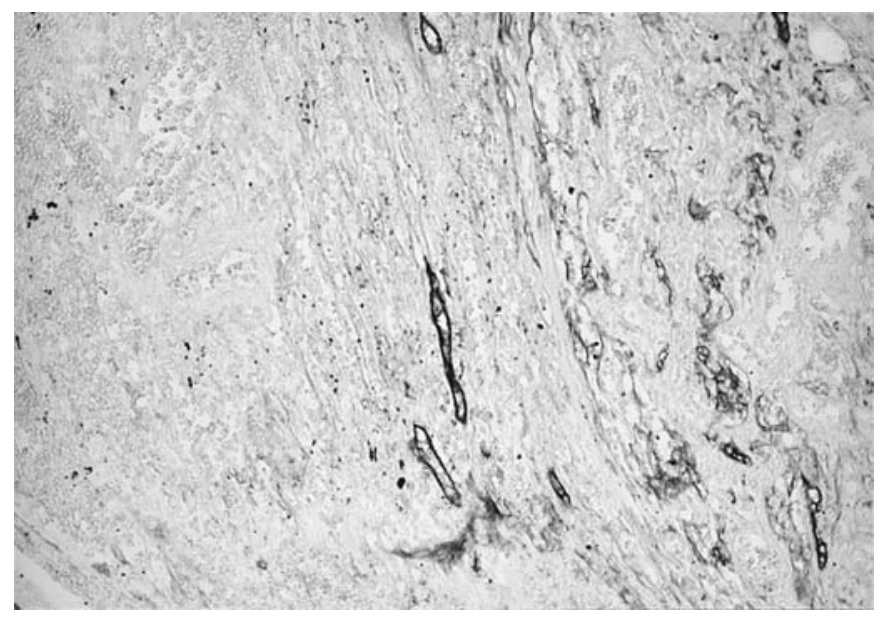

Figure 2. D2-40-positive lymphatic vessels. Original magnification, x37.5.

$10 \%$ normal rabbit serum. Overnight incubation with the anti-D2-40 monoclonal antibody (Signet Laboratories, Dedham, MA), anti-F8RA monoclonal antibody (Nichirei, Tokyo, Japan), anti-VEGF-C polyclonal antibody (Santa Cruz Biotechnology, CA) and anti-Ang-2 polyclonal antibody (Santa Cruz Biotechnology) was followed by incubation with biotinylated rabbit anti-mouse $\mathrm{IgG}$ and biotinylated rabbit anti-goat IgG, respectively, and an avidin-biotin-peroxidase reaction that used 3,3'-diaminobenzidine tetrahydrochloride in the presence of $0.05 \% \mathrm{H}_{2} \mathrm{O}_{2}$. Negative control sections were treated using nonimmunized mouse $\mathrm{IgG}$ and goat $\mathrm{IgG}$ as the primary antibodies. Normal pulmonary tissue sections were used as positive controls.

Specimen classification based on immunohistochemical results. Two independent observers without knowledge of the patient data simultaneously evaluated the immunohistochemical results. The lymphatic microvessel density and microvessel density were evaluated according to the methods previously described (7). Briefly, after immunostaining with anti-D2-40 antibody and anti-F8RA antibody, the stained sections of D2-40 and F8RA were screened at an original magnification of $\mathrm{x} 100$ to identify the regions of highest

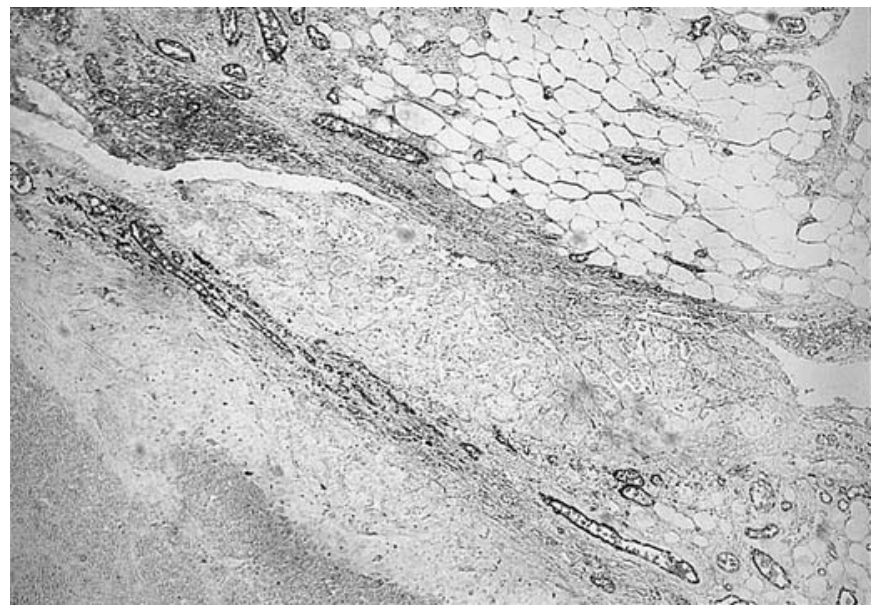

Figure 3. Some F8RA-positive vessels are found in the same tumor. Original magnification, $\mathrm{x} 15$.

lymphatic microvessel density and microvessel density. The lymphatic microvessel density and microvessel density were chosen at random and determined in different fields of serial sections. A grid $(0.15 \times 0.20 \mathrm{~mm})$ that defined areas of $0.03 \mathrm{~mm}^{2}$ per field was used to count the lymphatic vessels and microvessels at an original magnification of x400 (x40 objective lens with a $\mathrm{x} 10$ eyepiece) in three regions with the highest lymphatic microvessel density and microvessel density. The number of lymphatic vessels and microvessels was determined by averaging three separate counts of each grid made by two observers. The degree of VEGF-C reactivity within individual tissue sections was considered positive if unequivocal staining of carcinoma cells was seen in $>10 \%$ of the tumor cells; when $<10 \%$ were stained, the samples was classified as negative in VEGF-C, as previously described (8). For Ang-2 immunohistochemistry, tumor sections were considered positive if $>5 \%$ of tumor cells were immunoreactive, as previously described (9).

Statistical analysis. All data regarding the clinical and histopathologic variables were stored in a computer. The StatView program (Abacus Concepts, Berkeley, CA, USA) was used for all statistical analyses. Association between the clinicopathological data and expression of D2-40 immunohistochemical expression was analyzed using the Chi-square test and Student's t-test. A stepwise logistic regression model was used for the multivariate analysis. All tests were considered significant when $\mathrm{p}<0.05$.

\section{Results}

D2-40, F8RA, VEGF-C, and Ang-2 expression in NSCLC. The expression of D2-40 in the lymphatics was confirmed in the bronchial submucosa. The F8RA positive vascular network was slightly noted in alveolar tissues. D2-40 expression was mainly present in thin-walled lymphatic structures (Figs. 1 and 2). D2-40-positive lymphatic vessels were found in all cases. Lymphatic vessels were observed both within the tumor mass and in the peritumoral area or tumor edge. Lymphatic vessels in the peritumoral area or the tumor edge were more numerous and occupied a greater relative area compared with intratumoral lymphatics. The regions with the 


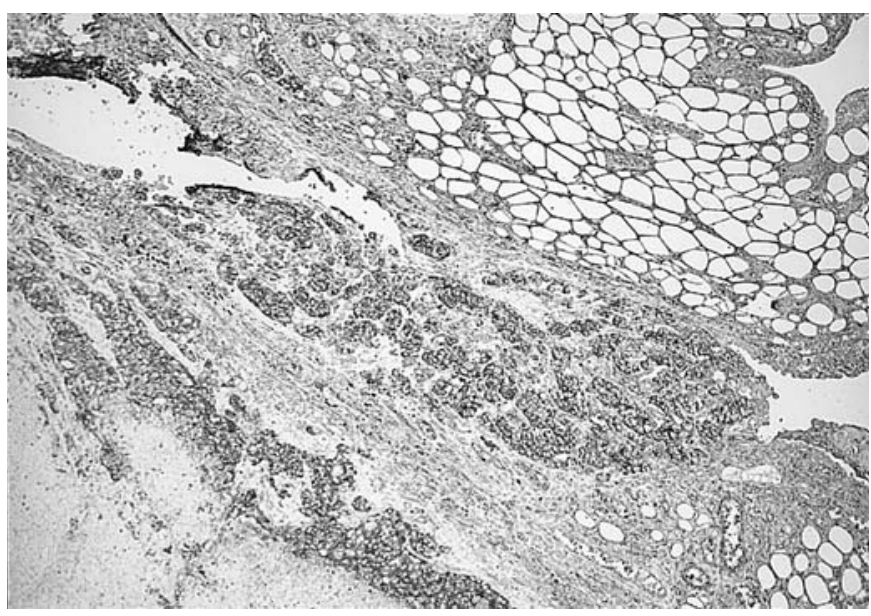

Figure 4. VEGF-C expression in carcinoma cells. Original magnification, $\mathrm{x} 15$.

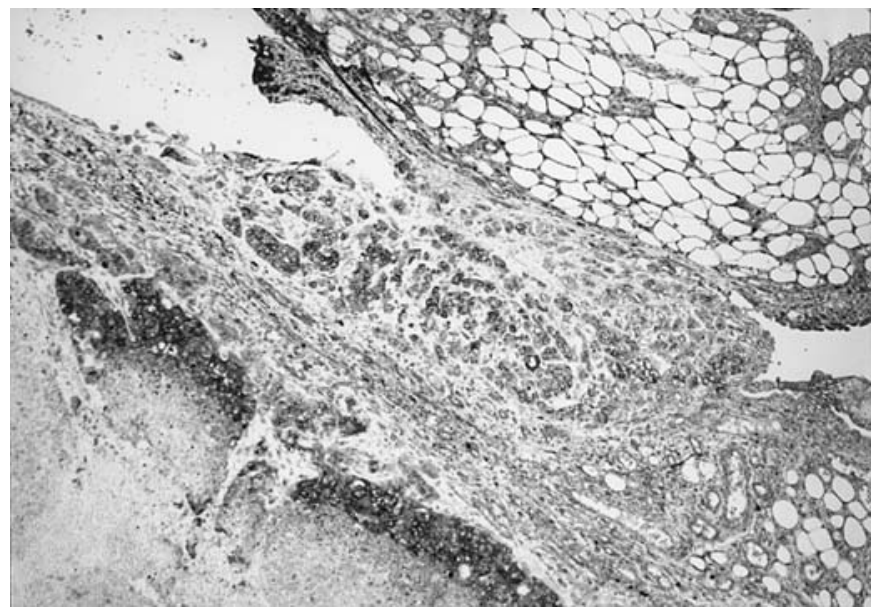

Figure 5. Ang-2 expression in carcinoma cells. Original magnification, x15.

greatest number of D2-40-positive vessels were most frequently the peritumoral area within $500 \mu \mathrm{m}$ from the tumor border or the margins of the tumor. The median number of D2-40-positive vessels in the highest lymphatic microvessel density was 25 (range, 5-71). The regions of greatest vascularization using F8RA were most frequently apparent at the margins of the tumors, as most of D2-40 vessels were consistently noted (Fig. 3). The median number of F8RApositive vessels was 15 (range, 5-52). Immunohistochemical assay of VEGF-C and Ang-2 revealed that VEGF-C and Ang-2 were expressed predominantly in cancer tissues (Figs. 4 and 5), and very low expression of VEGF-C and Ang-2 were present in normal tissues. Positive expression of VEGF-C and Ang-2 were primarily seen at the edges of tumor tissues and found in the cytoplasm of tumor cells. Of 77 cases, 46 patients $(58.7 \%)$ were positive for VEGF-C, and 26 patients (34.2\%) were positive for Ang-2.

Association between expression of D2-40, F8RA, VEGF-C, Ang-2 expression and clinicopathological factors. We classified the cases into two subgroups split by median D2-40 count: a low D2-40 density group $(n=39)$ for which D2-40 density was $\leq 25$; and a high D2-40 density group
Table I. Association between D2-40 density and various clinicopathological factors in patients with lung cancer.

\begin{tabular}{lccc}
\hline & $\begin{array}{c}\text { D2-40 } \\
\text { density low } \\
(\leq 25)(n=39)\end{array}$ & $\begin{array}{c}\text { D2-40 } \\
\text { density high } \\
(>25)(n=38)\end{array}$ & p-value \\
Variable & & & \\
Gender & 30 & 26 & \\
Male & 9 & 12 & 0.3420
\end{tabular}

Tumor status

T1

16

$\mathrm{T} 2$

18

19

T3

Nodal status

Negative

Positive

33

14

Stage

IA/IB
IIA/IIB
IIIA

29

9

5

19

$<0.0001$

Lymphatic invasion

Negative

Positive

16

Vascular invasion

Negative

24

15

Positive

15

0.0870

Histology

Adenocarcinoma

21

22

Squamous cell ca.

17

14

Large cell ca.

1

2

0.7258

\section{VEGF-C protein}

$\begin{array}{lrr}\text { Negative } & 22 & 9 \\ \text { Positive } & 17 & 29\end{array}$

0.0030

Ang-2 protein

Negative

30

Positive

9

$\mathrm{MVD}^{\mathrm{a}}$

$\begin{array}{llll}\text { Low }(\leq 15) & 29 & 14 & \\ \text { High }(>15) & 10 & 23 & 0.0029\end{array}$

${ }^{a}$ Microvessel density median, 15; ca, carcinoma.

$(n=38)$ for which D2-40 density was $>25$. Table I shows the clinicopathological parameters for these two groups. There were no significant intergroup differences regarding gender ratio, histology, vascular invasion, and Ang-2 protein. However, D2-40 density was significantly related to the tumor status, lymph node status, stage, lymphatic invasion, VEGF-C and microvessel density ( $p=0.0149$ for tumor 
Table II. Statistical associations between lymph node metastasis and examined variables.

\begin{tabular}{|c|c|c|c|c|}
\hline \multirow[b]{2}{*}{ Variables } & \multicolumn{2}{|c|}{ Lymph node metastasis } & \multirow[b]{2}{*}{$\begin{array}{l}\text { Univariate } \\
\text { p-value }\end{array}$} & \multirow[b]{2}{*}{$\begin{array}{c}\text { Multivariate } \\
\text { p-value }\end{array}$} \\
\hline & $\begin{array}{l}\text { Negative } \\
(\mathrm{n}=47)\end{array}$ & $\begin{array}{l}\text { Positive } \\
(\mathrm{n}=30)\end{array}$ & & \\
\hline \multicolumn{5}{|l|}{ Tumor status } \\
\hline $\mathrm{T} 1$ & 19 & 3 & & \\
\hline $\mathrm{T} 2$ & 19 & 18 & & \\
\hline $\mathrm{T} 3$ & 9 & 9 & 0.0010 & 0.4404 \\
\hline \multicolumn{5}{|c|}{ Lymphatic invasion } \\
\hline Negative & 29 & 5 & & \\
\hline Positive & 18 & 25 & 0.0003 & 0.0340 \\
\hline \multicolumn{5}{|c|}{ Vascular invasion } \\
\hline Negative & 30 & 9 & & \\
\hline Positive & 17 & 21 & 0.0077 & 0.4337 \\
\hline \multicolumn{5}{|c|}{ VEGF-C protein } \\
\hline Negative & 25 & 6 & & \\
\hline Positive & 22 & 24 & 0.0078 & 0.7024 \\
\hline \multicolumn{5}{|l|}{ Ang-2 protein } \\
\hline Negative & 35 & 16 & & \\
\hline Positive & 12 & 14 & 0.0952 & 0.7448 \\
\hline \multicolumn{5}{|l|}{ MVD } \\
\hline Low $(\leq 15)$ & 32 & 14 & & \\
\hline High $(>15)$ & 15 & 16 & 0.1023 & 0.2025 \\
\hline \multicolumn{5}{|l|}{ LMVD } \\
\hline Low $(\leq 25)$ & 33 & 6 & & \\
\hline High $(>25)$ & 14 & 14 & $<0.0001$ & 0.0070 \\
\hline
\end{tabular}

status; $\mathrm{p}<0.0001$ for nodal status; $\mathrm{p}<0.0001$ for stage; $\mathrm{p}=0.0153$ for lymphatic invasion; $\mathrm{p}=0.0030$ for VEGF-C, and $\mathrm{p}=0.0029$ for microvessel density).

Associations between lymph node metastasis and examined variables. The independent predictors of lymph node metastasis were determined using stepwise logistic regression analysis. As shown in Table II, T factor, lymphatic invasion, vascular invasion, VEGF-C protein and the lymphatic microvessel density were correlated with lymph node metastasis by univariate analysis. According to the multivariate analysis of these variables, lymphatic invasion and the lymphatic microvessel density proved to be the predictors of lymph node metastasis $(\mathrm{p}=0.0340$ for lymphatic invasion and $\mathrm{p}=0.0070$ for lymphatic microvessel density).

\section{Discussion}

NSCLC is a leading cause of morbidity and mortality among men and women in the U.S. (10). Despite major advances in cancer treatment in past 2 decades, the prognosis of patients with lung cancer has improved only minimally (10). Lymph node metastasis is one of the most powerful prognostic markers for resected NSCLC (11). Tumor cell spread via lymphatic capillaries is an early feature of many carcinomas. Evidence of the formation of lymphatic capillaries (lymphangiogenesis) has raised the possibility that cells within primary tumors can contribute actively to lymphatic dissemination through the induction of a lymphangiogenic process. Studies on lymphangiogenesis rely on the use of specific markers of lymphatic endothelial cells. D2-40 antibody was reported to detect a fixation-resistant epitope on a 40-kDa O-linked sialoglycoprotein expressed in lymphatic endothelium, but not blood vessels (2). D2-40 immunoreactivity appears to be an appropriate marker to assess lymphangiogenesis. Microvessel density, which presents an estimate for tumor angiogenesis, has been associated with metastatic spread and/or prognosis in individuals with NSCLC (12). However, the lymphatic microvessel density using D2-40 has not yet been widely evaluated.

In this study, most of the D2-40-positive vessels were found to be located in the peritumoral area or at the tumor edge. Previous studies present evidence that the center of tumors do not contain functional lymphatics, however, the lymphatic vessels at the tumor margins do facilitate lymphatic spread of tumor cells (13). The high lymphatic microvessel density at the margins of the tumor is thought to indicate the probability of lymphatic metastasis (13). D2-40 
immunoreactivity did not show any association with various clinicopathological tumor features including gender, vascular invasion, and histology in our study. On the other hand, high levels of D2-40 immunoreactivity were significantly associated with tumor status, advanced stage, the presence of lymphatic invasion and lymph node metastasis. Our results are concordant with a previous study reporting that D2-40 lymphatic microvessel density correlated with tumor stage and lymph node metastasis in breast carcinoma (14). Bono et al also reported the significant association between the number of Lyve-1-positive vessels and lymph node metastasis (15).

The lymphatic microvessel density using D2-40 did not show any association with the expression of Ang-2 protein, but the lymphatic microvessel density had a significant association with VEGF-C and the microvessel density in our study. VEGF-C, a novel VEGF member, has been found to introduce not only angiogenesis but also lymphangiogenesis via VEGF-R2 and VEGF-R3 (16). VEGF-C appears to be an important lymphangiogenic factor, and the correlation of VEGF-C expression with lymph node metastasis has been reported in many malignant tumors $(17,18)$. VEGF-C may exert a more powerful effect to enhance lymphangiogenesis and facilitate lymphatic dissemination of tumor cells. Lymphangiogenesis appears to frequently accompany angiogenesis (19). The discovery of Ang-1 and Ang-2 has provided novel and important insights into the molecular mechanisms of blood vessel formation. Ang-2 is required for vascular remodeling during angiogenesis. The remodeling phase is crucial for the proper organization of new vessels, and it seems likely that a similar remodeling step occurs in lymphangiogenesis. The Ang-2 knockout perinatal mouse showed defects in the overall organization of their lymphatic vasculature, and Ang-2 was thought to relate to lymphatic vessel formation or lymphangiogenesis (20). A correlation between lymph node involvement and Ang-2 expression was found in colon and breast carcinomas $(21,22)$, but is controversial (23). VEGF and/or VEGF-C expression was reported to be present in tumors overexpressing Ang-2 (22). Another study, however, presents that VEGF-C plays a role in the early stage of lymphatic vessel formation, and Ang-2 plays a role in the later remodeling stages (20). Our results that lymphatic microvessel density did not show an association with the expression of Ang-2 protein, but lymphatic microvessel density had a significant association with VEGF-C may be in accordance with results of this previous study. Lymphangiogenesis is often seen together with angiogenesis because newly emerging blood vessels are leaky and need lymphatic growth to avoid tissue edema (23). A significant correlation between lymphangiogenesis and angiogenesis was found in this study.

Tumor state, vascular invasion and VEGF-C protein were not detected as being independent factors for lymph node metastasis by multivariate analysis. Multivariate analysis demonstrated that lymphatic invasion and the lymphatic microvessel density were independent factors for lymph node metastasis, with the lymphatic microvessel density found to be the most powerful independent factor. As VEGF-C was dependent on the lymphatic microvessel density, VEGF-C may statistically interfere with the lymphatic microvessel density on lymph node, and VEGF-C was not an independent factor for lymph node metastasis. In our study, a significant difference in the rate of patient survival was detected between patients whose tumors had high lymphatic microvessel density compared to those whose tumors had low lymphatic microvessel density. Multivariate analysis demonstrated that the lymphatic microvessel density was an independent factor for overall survival (data not shown). But the number of patients in our study was small, and further studies may be needed to broadly evaluate these findings.

In conclusion, this study showed that the lymphatic microvessel density using D2-40 was elevated in advanced NSCLC, and this elevation is likely the result of elevated levels of expression of lymphangiogenic factors and their specific receptor. Tumors with a high D2-40 vessel density are associated with lymph node metastasis compared with tumors with a low density. In multivariate analysis, D2-40 was shown to be the predictor for lymph node metastasis. Further studies are required to fully understand the role of D2-40 in the lymphatic development in NSCLC, which may represent a future target for new therapeutic strategies.

\section{References}

1. Marks A, Sutherland DR, Bailey D, et al: Characterization and distribution of an oncofetal antigen (M2A antigen) expressed on testinal germ cell tumors. Br J Cancer 80: 569-578, 1999.

2. Kahn HJ and Marks A: A new monoclonal antibody, D2-40, for detection of lymphatic invasion in primary tumors. Lab Invest 82: $1255-1257,2002$.

3. Nisato RE, Tille JS and Pepper MS: Lymphangiogenesis and tumor metastasis. Thromb Haemost 90: 591-597, 2003.

4. Jeltsch M, Kaipainen A, Joukov V, et al: Hyperplasia of lymphatic vessels in VEGF-C transgenic mice. Science 276: 1423-1425, 1997.

5. Maisonpierre PC, Suri C, Jones PF, et al: Angiopoietin-2 is required for postnatal angiogenesis and lymphatic patterning, and only the latter role is resqued by angiopoietin-1. Dev Cell 3: 411-423, 2002.

6. Takahashi M, Yoshimoto T and Kubo H: Molecular mechanisms of lymphangiogenesis. Int J Hematol 80: 29-34, 2004.

7. Takanami I, Takeuchi $\mathrm{K}$ and Naruke M: Mast cell density is associated with angiogenesis and poor prognosis in pulmonary adenocarcinoma. Cancer 88: 2686-2692, 2000.

8. Hoar FJ, Chaudri S, Wadley MS and Stonelake PS: Coexpression of vascular endothelial growth factor C (VEGF-C) and c-erb B2 in human breast carcinoma. Eur J Cancer 39: 1698-1703, 2003.

9. Tanaka F, Ishikawa K, Yanagihara K, et al: Expression of angiopoietins and its clinical significance in non-small cell lung cancer. Cancer Res 62: 7124-7129, 2002.

10. Ginsberg R, Vokes E and Raben A: Non-small cell lung cancer. In: Cancer: Principles and Practice of Oncology. DeVitaV Jr, Helleman S and Rosenberg S (eds). Lippencott-Raven, Philadelphia, pp858-911, 1997

11. Naruke T, Goya T, Tsuchiya R and Suematsu K: Prognosis and survival in resected lung carcinoma based on the international staging system. J Thorac Cardiovasc Surg 96: 440-447, 1998.

12. Macchiarini P, Fontaini G, Hardlin MJ, Squantini F and Angellitti GA: Regulation of neovasculisation to metastasis of non-small cell lung cancer. Lancet 340: 145-146, 1992.

13. Padera TP, Kadambi A, DiThomaso E, et al: Lymphatic metastasis in the absent of functional intratumoral lymphatics. Science 296: 1883-1886, 2002.

14. Choi WWL, Lewis M, Lawson D, et al: Angiogenic and lymphangiogenic microvessel density in breast carcinoma: correlation with clinicopathologic parameters and VEGF-family gene expression. Modern Pathol 18: 143-152, 2005.

15. Bono P, Wasenius VM, Heikkila P, Lundin J, Jackson DG and Joensuu H: High Lyve-1 positive lymphatic vessel numbers are associated with poor outcome in breast cancer. Clin Can Res 10: 7144-7149, 2004. 
16. Cao Y, Linden P, Farnebo J, et al: Vascular endothelial growth factor C induces angiogenesis in vivo. Proc Acad Sci USA 85: 14389-14394, 1998.

17. Sendivy R, Beck-Managetta J, Haverkampf C, Battistutti W and Henigschnabel S: Expression of vascular endothelial growth factor-C correlates with lymphatic microvessel density and the nodal status in oral squamous cell cancer. Oral Pathol Med 32: 4555-4560, 2003.

18. Niki T, Iba S, Tokunou M, Yamada T, Matsuno Y and Hirohashi S: Expression of vascular endothelial growth factor A, B, C, and D and theirrelationship to lymph node status in lung adenocarcinoma. Clin Cancer Res 6: 2431-2439, 2000.

19. Alitalo K and Carmeliet P: Molecular mechanisms of lymphangiogenesis in health and disease. Cancer Cell 1: 219-227, 2002 .
20. Oki Y, Yasunaga K, Ito Y, et al: Angiopoietin-related growth factor (AGF) promotes epidermal proliferation, remodeling, and regeneration. Proc Natl Acad Sci USA 100: 9494-9499, 2003.

21. Ochiumi T, Tanaka S, Oka S, et al: Clinical significance of angiopoietin-2 expression at the deepest invasive tumor site of advanced colorectal carcinoma. Int J Oncol 24: 539-547, 2004.

22. Sfiligoi C, deLuca AD, Cascone I, et al: Angiopoietin-2 expression in breast cancer correlates with lymph node invasion and short survival. Int J Cancer 103: 466-474, 2003.

23. Sun XD, Liu XE, Wu JM, Cai XJ, Mou YP and Li JD: Expression and significance of angiopoietin-2 in gastric cancer. World J Gastroenterol 10: 1382-1385, 2004.

24. Thurston G: Role of angiopoietins and Tie receptor tyrosine kinases in angiogenesis and lymphangiogenesis. Cell Tissue Res 314: 61-68, 2003. 